\title{
Indecomposable Quasi-Characteristics Scheme on Pyramidal Stencil and Its Application for Numerical Simulation of Two-phase Flows Through Heterogeneous Porous Medium
}

\author{
Do Young Kwak, Mikhail P. Levin, and Sungyun Lee \\ Department of Mathematics \\ Korea Advanced Institute of Science and Technology \\ Taejon, 305-701, Republic of Korea
}

Received 27, December 2000; accepted 30 May 2001

\begin{abstract}
A new high-resolution indecomposable quasi-characteristics scheme with monotone properties based on pyramidal stencil is considered. This scheme is based on consideration of two high-resolution numerical schemes approximated governing equations on the pyramidal stencil with different kinds of dispersion terms approximation. Two numerical solutions obtained by these schemes are analyzed, and the final solution is chosen according to the special criterion to provide the monotone properties in regions where discontinuities of solutions could arise. This technique allows to construct the high-order monotone solutions and keeps both the monotone properties and the high-order approximation in regions with discontinuities of solutions. The selection criterion has a local character suitable for parallel computation. Application of the proposed technique to the solution of the time-dependent 2D two-phase flows through the porous media with the essentially heterogeneous properties is considered, and some numerical results are presented. (C) 2002 John Wiley \& Sons, Inc. Numer Methods Partial Differential Eq 18: 44-55, 2002
\end{abstract}

Keywords: quasi-characteristics; monotone schemes; porous media flows

\section{INTRODUCTION}

Numerical schemes of the method of characteristics have an essentially higher precision compared with other approaches because they properly take into account characteristic properties of governing equations of hyperbolic type. However, in modern practice these schemes are not widely used because of their complexity, especially in 3D case. To overcome the computational difficulties of the method of characteristics in [1-5] new quasi-characteristics schemes of vari-

Correspondence to: Mikhail P. Levin, Department of Mathematics, Korea Advanced Institute of Science and Technology, Taejon 305-701, Republic of Korea (e-mail: levin@math.kaist.ac.kr; Mikhail_Levin@ hotmail.com)

Contract grant sponsor: The BK21 project

(C) 2002 John Wiley \& Sons, Inc.

DOI 10.1002/num.1039 
ous types were proposed. These schemes in turn are a generalization of well-known backward characteristics schemes [6]. In our article we develop a new high-resolution indecomposable quasi-characteristics scheme with monotone properties on the pyramidal stencil. It is based on the approximation of the governing transport equations written in expanded characteristic form along quasi-characteristics (special grid lines forming a region containing characteristics of governing equations and lying at a short distance with respect to characteristics). We take ribs of pyramidal stencil as quasi-characteristics. To provide monotone properties of solutions two high-resolution schemes with different dispersion properties are taken into account. For the selection of the final solution a special heuristic criterion is proposed. This scheme is applied to the solution of transport equation in the coupled system of equations [7-9] describing the time-dependent, 2D two-phase flows through the porous media with the essentially heterogeneous properties. Some numerical results shown the efficiency of considering approach are presented.

\section{INDECOMPOSABLE QUASI-CHARACTERISTICS SCHEMES ON THE PYRAMIDAL STENCIL}

Let us construct the indecomposable quasi-characteristics schemes on the pyramidal stencil for the $3 \mathrm{D}$ transport equation

$$
\frac{\partial u}{\partial t}+b_{1} \frac{\partial u}{\partial x}+b_{2} \frac{\partial u}{\partial y}=b_{3}, \quad-\infty<(x, y)<+\infty, \quad 0<t \leq T
$$

satisfying the following initial conditions:

$$
u(0, x, y)=u_{0}(x, y)
$$

Here $u(t, x, y)$ is a searching function, and $b_{i}=b_{i}\left(t, x, y, u, \frac{\partial u}{\partial x}, \frac{\partial u}{\partial y}\right), \quad(i=1,2,3)$, and $u_{0}(x, y)$ are data known functions.

The first step of the quasi-characteristics technique consists in transformation of the governing Eq. (2.1) into the expanded characteristics form along any spatial line $l$ in $(t, x, y)$ space as follows:

$$
\frac{d u}{d t}+\left[b_{1}-\left(\frac{d x}{d t}\right)_{l}\right] \frac{\partial u}{\partial x}+\left[b_{2}-\left(\frac{d y}{d t}\right)_{l}\right] \frac{\partial u}{\partial y}=b_{3}
$$

Here $\frac{d u}{d t}$ is a total derivative of the searching function $u$ with respect to $t$ along any line $l$.

Approximating the expanded characteristic form of the governing equation along different lines $l$ we shall construct a numerical scheme similar to the well-known backward characteristic scheme [6].

Let us consider a finite difference grid, for simplicity, uniform in each direction in space $(t, x, y)$ with steps $\tau, h_{x}$ and $h_{y}$, and a pyramidal stencil $P_{1} P_{2} P_{3} P_{4} R$ in the grid space (Fig. 1 ). The basement of this stencil $P_{1} P_{2} P_{3} P_{4}$ belongs to some data layer $t=t_{0}$ and the vertex $\mathrm{R}$ belongs to the new layer $t=t_{0}+\tau$. Coordinates of the above mentioned vertices are as follows: $P_{1}\left(t_{0}, x_{0}+h_{x}, y_{0}-h_{y}\right), P_{2}\left(t_{0}, x_{0}+h_{x}, y_{0}+h_{y}\right), P_{3}\left(t_{0}, x_{0}-h_{x}, y_{0}+h_{y}\right), P_{4}\left(t_{0}, x_{0}-h_{x}, y_{0}-h_{y}\right)$, $R\left(t_{0}+\tau, x_{0}, y_{0}\right)$. Also we take into consideration $m_{0}\left(t_{0}, x_{0}, y_{0}\right)$ a center point of the basement of the pyramid stencil and denote the nodal points corresponding to the central points of the pyramid basement ribs as follows: $m_{+}\left(t_{0}, x_{0}+h_{x}, y_{0}\right), m_{-}\left(t_{0}, x_{0}-h_{x}, y_{0}\right), n_{+}\left(t_{0}, x_{0}, y_{0}+h_{y}\right)$, $n_{-}\left(t_{0}, x_{0}, y_{0}-h_{y}\right)$. 


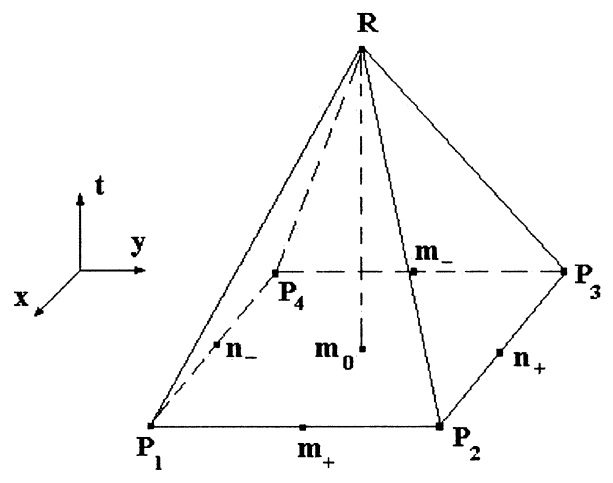

FIG. 1. The pyramidal stencil.

Approximating Eq. (2.3) along ribs $P_{i} R$, we obtain

$$
\begin{aligned}
\frac{u_{R}-u_{P_{i}}}{\tau}+\left[\left(b_{1}\right)_{P_{i} R}-\frac{x_{R}-x_{P_{i}}}{\tau}\right]\left(\frac{\partial u}{\partial x}\right)_{P_{i} R} & \\
& +\left[\left(b_{2}\right)_{P_{i} R}-\frac{y_{R}-y_{P_{i}}}{\tau}\right]\left(\frac{\partial u}{\partial y}\right)_{P_{i} R}=\left(b_{3}\right)_{P_{i} R},
\end{aligned}
$$

where $i=1,2,3,4$.

According to [4] we take the approximation of the outward derivatives at the middle layer $t=t_{0}+\frac{\tau}{2}$ :

$$
\begin{aligned}
& \left(\frac{\partial u}{\partial x}\right)_{t=t_{0}+\frac{\tau}{2}}=\left(\frac{\partial u}{\partial x}\right)_{C}+\left(x-x_{0}\right) W+d\left(y-y_{0}\right), \\
& \left(\frac{\partial u}{\partial y}\right)_{t=t_{0}+\frac{\tau}{2}}=\left(\frac{\partial u}{\partial y}\right)_{C}+\left(y-y_{0}\right) V+d\left(x-x_{0}\right) .
\end{aligned}
$$

\section{A. Scheme I}

In this case we take a center point of the middle section of the pyramidal stencil $\left(t_{0}+\frac{\tau}{2}, x_{0}, y_{0}\right)$ as the point $C$ (or $C_{I}$ ) and choose values $W$ and $V$ (or $W_{I}$ and $V_{I}$ ) at the middle layer $t_{0}+\frac{\tau}{2}$ by the following formulas:

$$
\begin{gathered}
W_{I}=W\left(m_{0}\right) \equiv \frac{1}{3}\left[\frac{u_{P_{1}}-2 u_{n_{-}}+u_{P_{4}}}{h_{x}^{2}}\right. \\
\left.+\frac{u_{m_{+}}-2 u_{m_{0}}+u_{m_{-}}}{h_{x}{ }^{2}}+\frac{u_{P_{2}}-2 u_{n_{+}}+u_{P_{3}}}{h_{x}{ }^{2}}\right], \\
V_{I}=V\left(m_{0}\right) \equiv \frac{1}{3}\left[\frac{u_{P_{2}}-2 u_{m_{+}}+u_{P_{1}}}{h_{y}{ }^{2}}\right. \\
\left.+\frac{u_{n_{+}}-2 u_{m_{0}}+u_{n_{-}}}{h_{y}{ }^{2}}+\frac{u_{P_{3}}-2 u_{m_{-}}+u_{P_{4}}}{h_{y}{ }^{2}}\right] .
\end{gathered}
$$

Here $W\left(m_{0}\right)$ and $V\left(m_{0}\right)$ are finite difference operators approximating the second-order derivatives of the searching function with the second-order approximation error on the appropriate stencil with middle point $m_{0}$. 
Substitution of (2.5-2.8) into (2.4) yields a system of four equations for evaluation of $u_{R}$, $\left(\frac{\partial u}{\partial y}\right)_{C},\left(\frac{\partial u}{\partial y}\right)_{C}$, and $d$. Solving it, we obtain $u_{R}^{I}$. In the nonlinear case in the above-mentioned algorithm, it is necessary to do three iterations as in the usual method of characteristics.

\section{B. Scheme II}

To construct a second-order scheme with different dispersive properties, we choose the point $C_{I I}$ and values $W_{I I}, V_{I I}$ according to the following algorithm:

$$
\begin{gathered}
\text { if } b_{1}\left(x_{0}, y_{0}, t_{0}\right) \geq 0 \text { and } b_{2}\left(x_{0}, y_{0}, t_{0}\right) \geq 0 \\
\text { then } W_{I I}=W\left(P_{4}\right), V_{I I}=V\left(P_{4}\right), C_{I I}=\left(P_{4 x}, P_{4 y}, t_{0}+\frac{\tau}{2}\right), \\
\text { if } b_{1}\left(x_{0}, y_{0}, t_{0}\right) \geq 0 \text { and } b_{2}\left(x_{0}, y_{0}, t_{0}\right)<0 \\
\text { then } W_{I I}=W\left(P_{3}\right), V_{I I}=V\left(P_{3}\right), C_{I I}=\left(P_{3 x}, P_{3 y}, t_{0}+\frac{\tau}{2}\right) \\
\text { if } b_{1}\left(x_{0}, y_{0}, t_{0}\right)<0 \text { and } b_{2}\left(x_{0}, y_{0}, t_{0}\right)<0 \\
\text { then } W_{I I}=W\left(P_{2}\right), V_{I I}=V\left(P_{2}\right) C_{I I}=\left(P_{2 x}, P_{2 y}, t_{0}+\frac{\tau}{2}\right) \\
\text { if } b_{1}\left(x_{0}, y_{0}, t_{0}\right)<0 \text { and } b_{2}\left(x_{0}, y_{0}, t_{0}\right) \geq 0 \\
\text { then } W_{I I}=W\left(P_{1}\right), V_{I I}=V\left(P_{1}\right), C_{I I}=\left(P_{1 x}, P_{1 y}, t_{0}+\frac{\tau}{2}\right) .
\end{gathered}
$$

Substitution of (2.5), (2.6), and (2.9) into (2.4) yields a system of four equations for evaluation of $u_{R},\left(\frac{\partial u}{\partial x}\right)_{C},\left(\frac{\partial u}{\partial y}\right)_{C}$, and $d$. Solving it, we obtain $u_{R}^{I I}$. As it was mentioned in previous subsection that in nonlinear case, it is necessary to do three iterations in evaluation of $u_{R}^{I I}$.

\section{Selection Criterion}

As it was shown in [1,2], it is possible to construct the final solution with monotone properties by choosing of one of two admissible solutions of the second-order approximation with different dispersive properties. The appropriate criterion proposed in cited articles is based on the analysis of the average value of the governing transport equation operator evaluated on each elementary mesh cell by the high-order quadrature formulas. This criterion takes into consideration the history of computations in previous grid points and therefore is not suitable for the parallel computations and multidimensional case. In [5] a simpler heuristic criterion based on the minimization of the rough approximation of the average value of governing operator was proposed. This criterion does not take into account the history of computations, has a local character, and is suitable for the parallel realization. In this article, we construct a simple heuristic criterion as a minimal principle for the increment of searching function over the stencil in following form:

$$
u_{R}^{\text {final }}=\min _{i=I, I I}\left|u_{R}^{i}-\frac{C_{0} u_{m_{0}}+C_{1} u_{P_{1}}+C_{2} u_{P_{2}}+C_{3} u_{P_{3}}+C_{4} u_{P_{4}}}{C_{0}+C_{1}+C_{2}+C_{3}+C_{4}}\right| .
$$

Here $C_{i}, i=0,1,2,3,4$ are some constants to be chosen. As our numerical tests show, the best result corresponds to the following set: $C_{0}=1, C_{i}=0, i=1,2,3,4$.

Thus, the final solution in each grid point is chosen among two admissible solutions $u_{R}^{I}$ and $u_{R}^{I I}$ according to the following simple minimal principle:

$$
u_{R}^{\text {final }}=\min _{i=I, I I}\left|u_{R}^{i}-u_{m_{0}}\right| .
$$


It is easy to see that this principle has a local character, and it is very suitable for parallel computations, because it allows in principle to provide computations of searching function in each grid node independently in separate processors in computers with massive parallel processors, and in computers with pipeline processors it allows to provide the maximal loading of pipeline.

\section{TWO-PHASE POROUS MEDIA FLOWS (OIL AND WATER)}

\section{A. Governing Equations}

Let us apply the algorithm described above to the numerical simulation of two-phase flows through essentially heterogeneous porous medium. The governing equations according [6-8] can be presented in the following form with respect to the water saturation $s$ and the pressure $p$ as unknown functions:

$$
\begin{gathered}
m\left(\frac{\partial s}{\partial t}\right)-\frac{\partial}{\partial x}\left(\frac{k k_{w}}{\mu_{w}} \frac{\partial p}{\partial x}\right)-\frac{\partial}{\partial y}\left(\frac{k k_{w}}{\mu_{w}} \frac{\partial p}{\partial y}\right)=0, \\
\frac{\partial}{\partial x}\left[k\left(\frac{k_{w}}{\mu_{w}}+\frac{k_{o}}{\mu_{o}}\right) \frac{\partial p}{\partial x}\right]+\frac{\partial}{\partial y}\left[k\left(\frac{k_{w}}{\mu_{w}}+\frac{k_{o}}{\mu_{o}}\right) \frac{\partial p}{\partial y}\right]=0 .
\end{gathered}
$$

Here $m$ is a porosity, $k=k(x, y)$ is an absolute penetration of porous medium, $k_{w}=k_{w}(s)$ and $k_{o}=k_{o}(s)$ are a relative penetration of water and oil, and $\mu_{w}$ and $\mu_{o}$ are a viscosity of water and oil. Let us notice that the oil saturation $s_{O}$ can be evaluated by the water saturation according to the following simple formula: $s_{o}=1-s$.

Because $k_{w}$ and $k_{o}$ are functions only of water saturation $s$, then Eq. (3.1) could be presented in the form (2.1) as follows

$$
\frac{\partial s}{\partial t}-\left(\frac{k}{m \mu_{w}} \frac{\partial p}{\partial x} \frac{d k_{w}}{d s}\right) \frac{\partial s}{\partial x}-\left(\frac{k}{m \mu_{w}} \frac{\partial p}{\partial y} \frac{d k_{w}}{d s}\right) \frac{\partial s}{\partial y}=\frac{k_{w}}{m}\left[\frac{\partial}{\partial x}\left(\frac{k}{\mu_{w}} \frac{\partial p}{\partial x}\right)+\frac{\partial}{\partial y}\left(\frac{k}{\mu_{w}} \frac{\partial p}{\partial y}\right)\right] .
$$

We can see that the system of governing equations (3.2-3.3) has a mixed type. Equation (3.3) is a nonlinear transport equation of hyperbolic type, and Eq. (3.2) has the elliptic type. Further, we could consider the transport Eq. (3.3) as a governing equation and the elliptic Eq. (3.2) as a nonlinear restriction for coefficient of the governing equation. Then we could apply the quasicharacteristics technique described in the previous section to solve the initial boundary-value problem for the transport equation, and also on each time level we need to solve the boundary value problem for elliptic equation to define the coefficients of the governing equation. In our approach for the solution of the boundary value problem for the elliptic equation we used the wellknown five-points finite difference conservative scheme and bi-conjugate gradient algorithm as in [7-9].

\section{B. Initial and Boundary Conditions}

Let us consider rectangle flow regions $D=0 \leq x \leq L, 0 \leq y \leq H$ consisting of two subregions $D_{1}=0.2 L \leq x \leq 0.8 L, 0 \leq y \leq \frac{H}{2}$ and $D_{2}=D-D_{1}$ shown in Fig. 2.

Let us suppose that the absolute penetration $k$ in the flow region is defined as piecewise constant function as follows: 


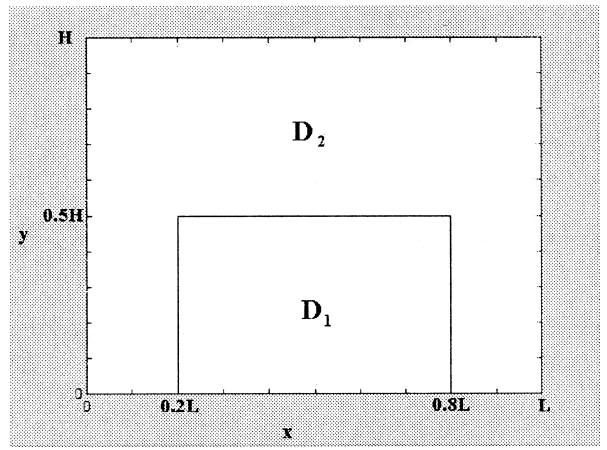

FIG. 2. Flow region.

$$
k= \begin{cases}k_{D_{1}}, & \text { if }(x, y) \in D_{1}, \\ k_{D_{2}}, & \text { if }(x, y) \in D_{2} .\end{cases}
$$

For the transport Eq. (3.3) we set the following initial conditions:

$$
s(x, y, 0)=\left\{\begin{array}{l}
0.2, \text { if } 0 \leq x<L, 0 \leq y \leq H, \\
1.0, \text { if } x=L, 0 \leq y \leq H
\end{array}\right.
$$

and also boundary conditions as follows:

$$
\begin{gathered}
\frac{\partial s}{\partial y}=0, \text { if } t>0, y=0, H, 0 \leq x \leq L ; \\
s(L, y, t)=1.0 \text {, if } 0 \leq y \leq H, t>0 .
\end{gathered}
$$

For the pressure Eq. (3.2) we set the following boundary conditions:

$$
\begin{gathered}
\frac{\partial p}{\partial y}=0, \text { if } 0<x<L, y=0, H \\
p=P_{0}, \text { if } x=0,0 \leq y \leq H \\
\frac{\partial p}{\partial x}=\frac{Q_{0} \mu_{w}}{H k k_{w}}, \text { if } x=L, 0 \leq y \leq H .
\end{gathered}
$$

Here $P_{0}$ and $Q_{0}$ are known parameters.

The relative penetrations of the water $k_{w}$ and oil $k_{o}$ are chosen as follows:

$$
\begin{gathered}
k_{w}(s)=\left\{\begin{array}{c}
0, \text { if } s \leq 0.1 ; \\
\left(\frac{s-0.1}{0.7}\right)^{3}, \quad \text { if } 0.1<s \leq 0.8 ; \\
1, \text { if } s>0.8 ;
\end{array}\right. \\
k_{o}(s)=\left\{\begin{array}{c}
1, \text { if } s \leq 0.1 ; \\
\left(\frac{0.8-s}{0.7}\right)^{3}, \text { if } 0.1<s \leq 0.8 ; \\
0, \text { if } s>0.8 .
\end{array}\right.
\end{gathered}
$$



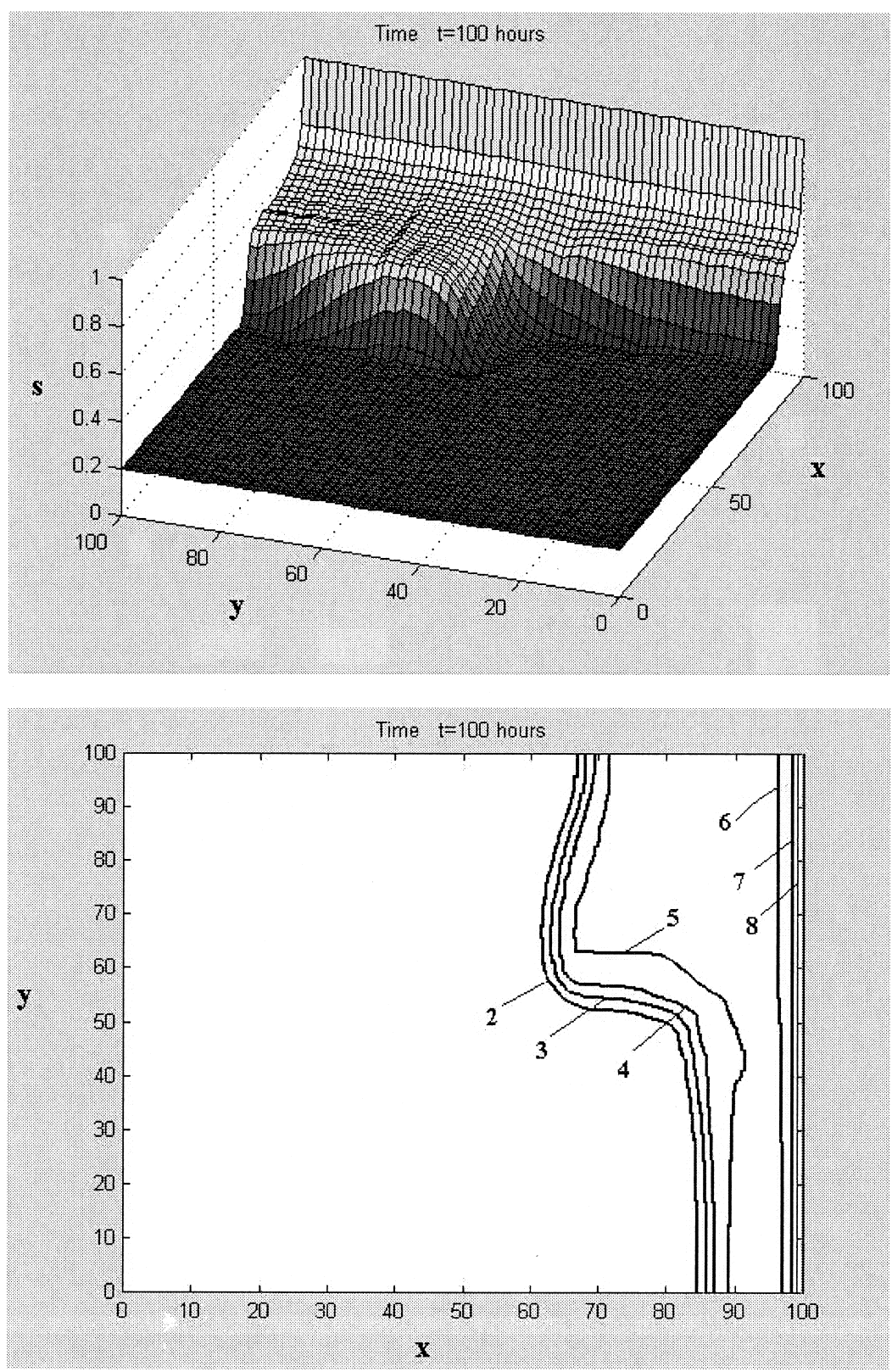

FIG. 3. The water saturation at $t=100$ hours.

Let us mention that the considering initial boundary value problem describes two-phase porous media flows between two horizontal wells where the left boundary $x=0$ corresponds to the production well and the right boundary $x=L$ corresponds to the injection well. 

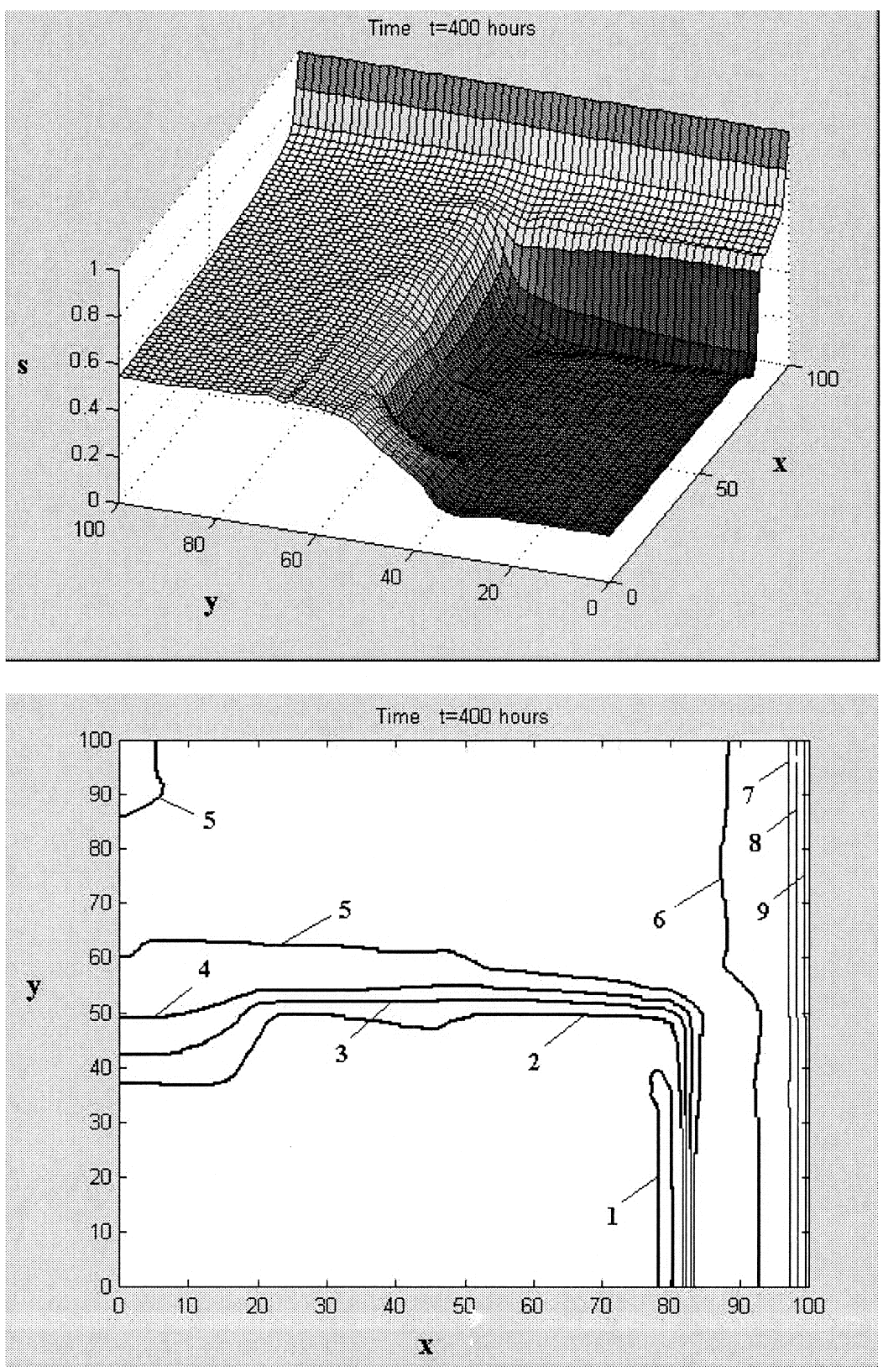

FIG. 4. The water saturation at $t=400$ hours.

\section{Numerical Results}

Now we consider some numerical results obtained for the following values of parameters $m=0.2$, $k_{D_{1}}=0.1 \cdot 10^{-12} \mathrm{~m}^{2}, k_{D_{2}}=1.0 \cdot 10^{-12} \mathrm{~m}^{2}, \mu_{w}=1 \cdot 10^{6} \mathrm{~N} \cdot \mathrm{sec} \cdot \mathrm{m}^{-2}, \mu_{o}=3 \cdot 10^{6} \mathrm{~N} \cdot \mathrm{sec} \cdot \mathrm{m}^{-2}$, $L=H=100 \mathrm{~m}, P_{0}=0$, and $Q_{0}=0.69444 \cdot 10^{-12} \mathrm{~m}^{2} \cdot \mathrm{sec}^{-1}$. Thus, the absolute penetration 

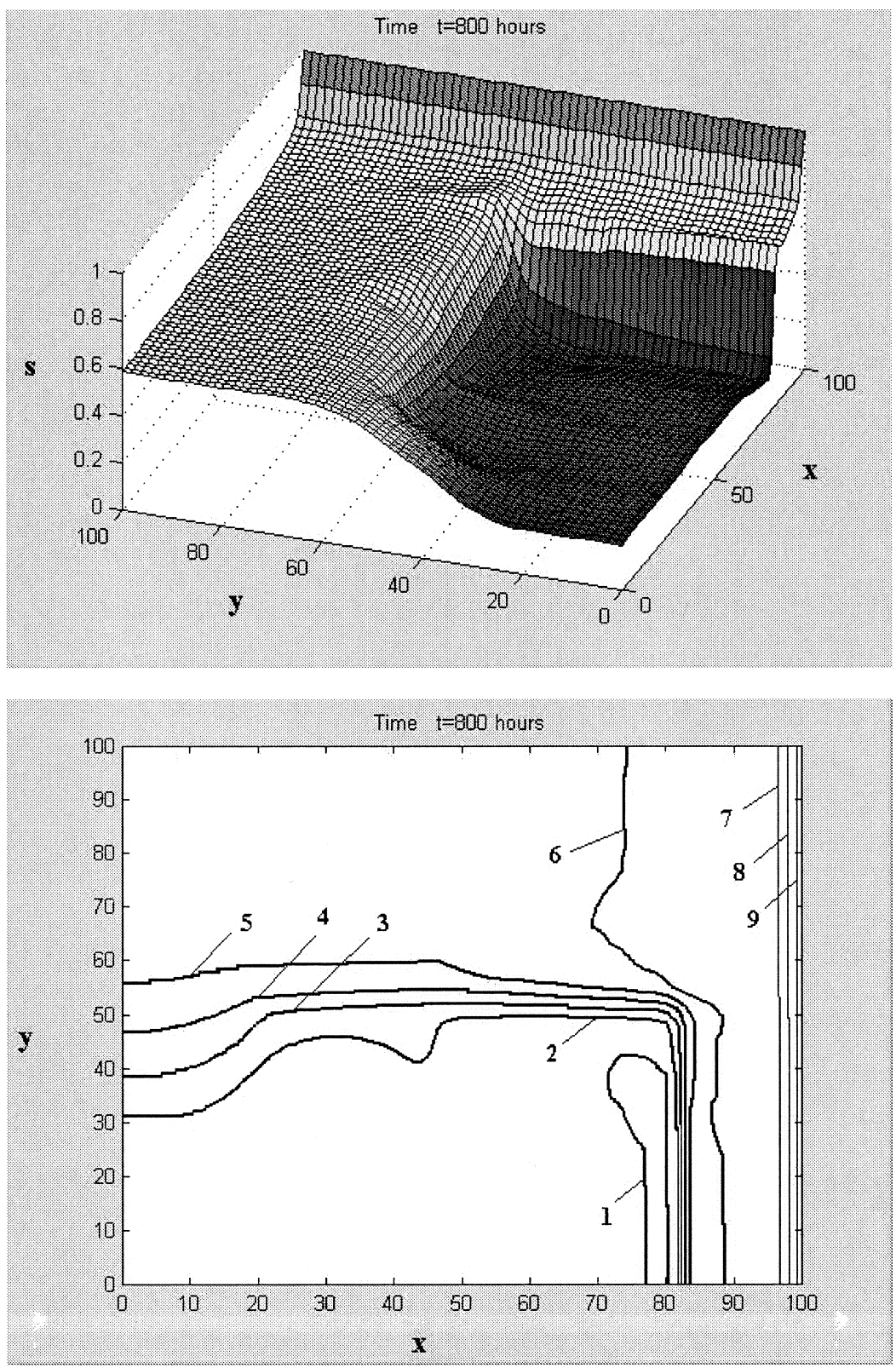

FIG. 5. The water saturation at $t=800$ hours.

in the subregion $D_{1}$ is 10 times less than those in subregion $D_{2}$. Computations were carried out on the uniform grid with $61 * 61$ nodal points in $(\mathrm{x}, \mathrm{y})$-space.

In Fig. 3 isolines of water saturation $s$ and appropriate 3D chart are presented for time $t=100$ hours. In Figs. 4, 5, and 6 the same results are shown for moments $t=400$ hours, $t=800$ hours, and $t=1200$ hours, respectively. Line 1 on these figures corresponds to $s=0.15$, line 2 to 

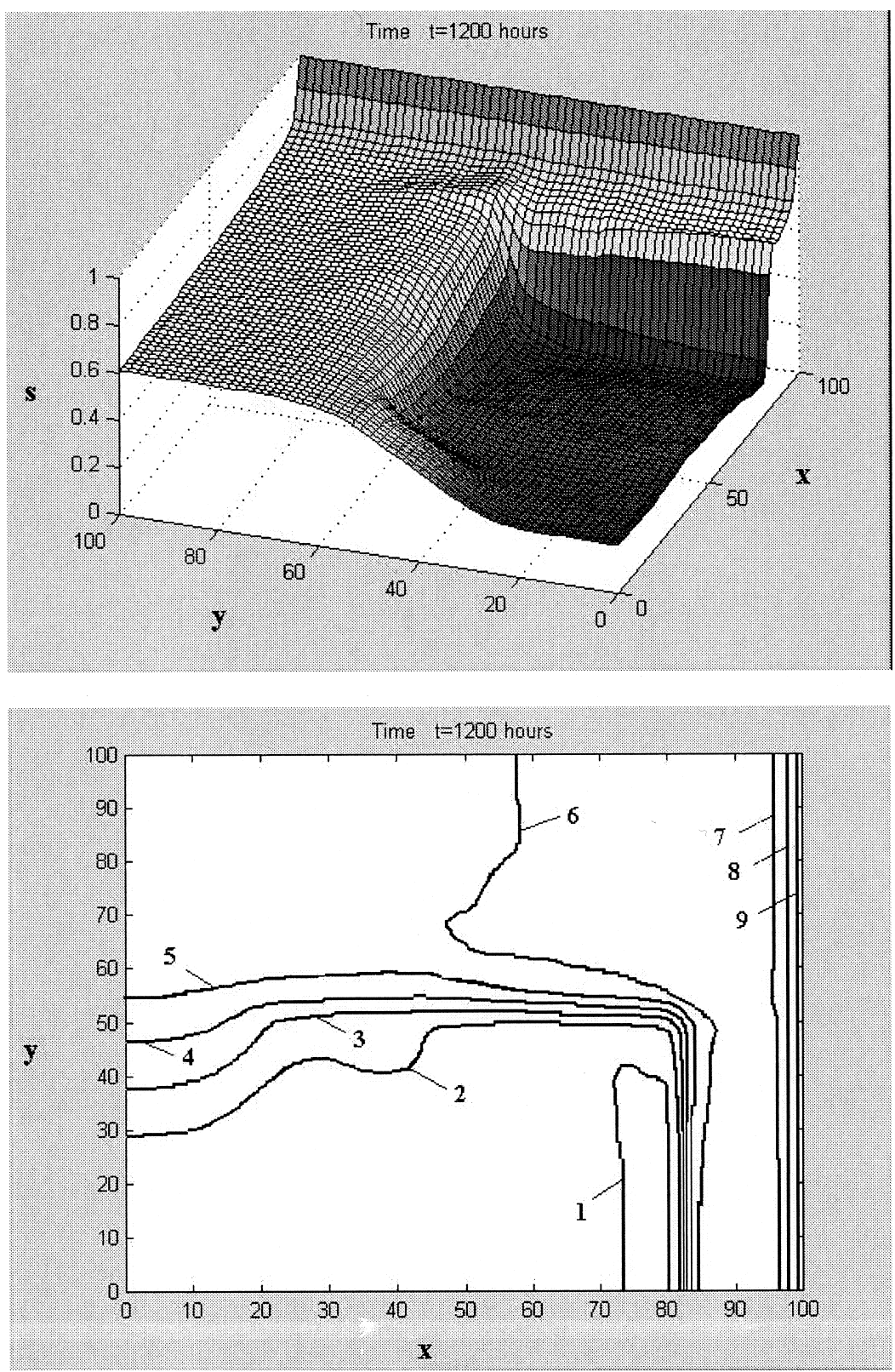

FIG. 6. The water saturation at $t=1200$ hours.

$s=0.25$, line 3 to $s=0.35$, line 4 to $s=0.45$, line 5 to $s=0.55$, line 6 to $s=0.65$, line 7 to $s=0.75$, line 8 to $s=0.85$, and line 9 to $s=0.95$.

In Fig. 7 two functions characterizing the efficiency of the oil recovery process by the water drive are shown. Line 1 corresponds to the ratio of the recovery oil to the total oil volume in initial moment $t=0$ with respect to time 


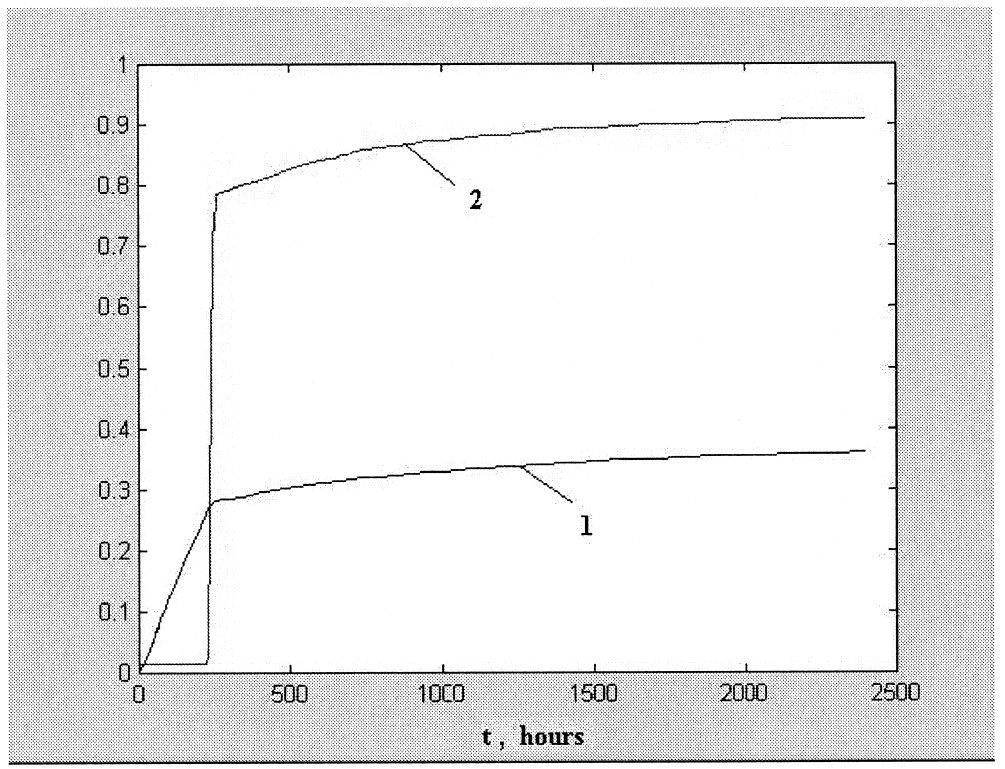

FIG. 7. Characteristics of efficiency of oil recovery. Line $1-\theta(t)$, line $2-\gamma(t)$.

$$
\theta(t)=\frac{\int_{D}[1-s(x, y, t)] d x d y}{\int_{D}[1-s(x, y, 0)] d x d y}
$$

and line 2 corresponds to the function

$$
\gamma(t)=\frac{\int_{0}^{L}\left[k \frac{k_{w}}{\mu_{w}} \frac{\partial p}{\partial x}\right]_{x=0} d y}{\int_{0}^{L}\left[k\left(\frac{k_{w}}{\mu_{w}}+\frac{k_{o}}{\mu_{o}}\right) \frac{\partial p}{\partial x}\right]_{x=0} d y}
$$

describing the water content in the development mixture at the production well corresponding to the boundary $x=0$.

According to the presented results, we can see that the solution of the considering problem has a wave type and the front of water wave solution is mainly spreading in the upper part of the flow region $D_{2}$ with high penetration. Subregion $D_{1}$ with low penetration plays the role of obstacle and prevents the spreading of water wave. The water wave is stopped at the right boundary of this subregion $x=0.8 L$ and because of the partial penetration of this subregion, the oil barrier-layer (the region restricted by line 1 in Figs. 3-6) is generated on the left side of the right boundary of the low penetration subregion. After the moment $t=250$ hours, when the water wave in the upper part of the flow region is close to the production well (boundary $x=0$ ), the efficiency of the oil recovery process falls down, and oil from the low penetration subregion and even from the high-penetration subregion $(0 \leq x \leq 0.2 L, 0 \leq y \leq 0.5 H)$, shaded by the first, almost can not be developed by the water drive. 
According to our results, we see that in the case considered in this article, it is possible to develop only about 35 percent of oil by the usual water drive technology although 70 percent of oil is contained in the high-penetration subregion. These results are in good correspondence with well-known practice.

\section{CONCLUSIONS}

Presented results show that numerical solution computed by the proposed algorithm has wave type and is free of the spurious oscillations near the wave fronts typical for pure high-order schemes and could deliver fine solutions on rough meshes. Therefore, this method could be efficiently applied for fast and exact modeling of practical porous media problems.

\section{References}

1. E. V. Nikolaevskaya, One class of running finite difference schemes, Computing Centre of USSR Academy of Sciences, Moscow, 1987.

2. V. M. Borisov, Yu. V. Kurilenko, I. E. Mikhailov, and E. V. Nikolaevskaya, A method of characteristics for calculation of vortex spatial supersonic stationary flows, Computing Centre of USSR Academy of Sciences, Moscow, 1988.

3. M. P. Levin, A difference scheme of quasi-characteristics and its use to calculate supersonic gas flows, J Comput Math Math Phys Russ Acad Sci 33 (1993), 113-121.

4. M. P. Levin, Quasi-characteristics numerical schemes, Hyperbolic problems: theory, numerics, application, Seventh International Conference in Zürich, February 1998, Vol. II, Birkhäuser Verlag, Basel, International Series on Numerical Mathematics 130, 1999, 619-628.

5. D. Y. Kwak and M. P. Levin, High resolution monotone scheme based on quasi-characteristics technique, Numer Meth Part Differ Eq 17 (2001), 262-276.

6. P. I. Chushkin, Numerical method of characteristics for three-dimensional supersonic flows, Progress in aeronautical sciences, Vol. 9, Pergamon Press, 1968, 41-122.

7. M. Yu. Zheltov and M. P. Levin, Application of the quasi-characteristics scheme for the two-phase flows through porous media, CFD J 2 (1993), 363-370.

8. A. I. Ibragimov, M. P. Levin, and L. V. Sidorov, Numerical investigation of two-phase fluid afflux to horizontal well by quasi-characteristics scheme, CFD J 8 (2000), 556-560.

9. A. I. Ibragimov, M. P. Levin, and L. V. Sidorov, Numerical investigation of high permeability thin layers influence on water filling of horizontal wells by quasi-characteristics scheme, CFD J 9 (2000), $105-109$. 